\title{
COLOCACIONES VERBALES INGLESAS. TRATAMIENTO LEXICOGRÁFICO ${ }^{* 1}$
}

\author{
Inés Lareo Martín ${ }^{* * *}$
}

Resumen: La oferta lexicográfica ha cambiado substancialmente desde hace algunos años, pero todavía presenta algunas carencias en cuanto a la inclusión de información sobre combinatoria léxica. Algunos autores consideran que esta información forma parte del significado de un lexema y, en consecuencia, debería estar incluida en su descripción lexicográfica.

Dado que compartimos esta opinión, hemos decidido examinar el contenido de algunos diccionarios monolingües, como el Oxford English Dictionary (1994), Longman Dictionary of Contemporary English (1995) y Collins COBUILD English Language Dictionary (1993). El análisis se centrará en la búsqueda de colocaciones formadas con un verbo light como make, have, take y do, seguido de un sustantivo.

Palabras clave: lexicografía, colocación, diccionarios, verbos soporte

Abstract: Though the lexicographic panorama has changed in the last few years, it still lacks some information about lexical combinations. Some authors are of the opinion that this information is part of the lexeme's meaning and, consequently, should be included in its lexicographic description.

As we share this opinion, we have decided to examine some of the English dictionaries, such as the Oxford English Dictionary (1994), Longman Dictionary of Contemporary English (1995) and Collins COBUILD English Language Dictionary (1993). The analysis will be focused on those collocations formed by a light verb, such as make, have, take or do, followed by a noun.

Keywords: lexicography, collocation, dictionaries, support verbs

\section{INTRODUCCIÓN}

El diccionario puede ser considerado como la caja fuerte del léxico. Los hablantes no tenemos la capacidad memorística suficiente para acordarnos de todos los lexemas ${ }^{2}$ de una

\footnotetext{
Fecha de recepción: marzo 2005

Fecha de aceptación y versión final: abril 2005

** Estudiante de doctorado, Departamento de Filología Inglesa, Universidad de A Coruña; alexines@udc.es.

${ }^{1}$ Este trabajo ha sido realizado, en parte, gracias a una beca de investigación concedida por la Xunta de Galicia.

${ }^{2}$ Utilizaremos este término según MeL'CuK (1988: 168), es decir, consideraremos lexema a una palabra utilizada en un sólo sentido. La diferenciación de los sentidos (o lexemas) encerrados en un lema es muy importante a la hora de hablar de combinatoria léxica, porque será ese sentido específico el que decidirá, en muchos casos, el verbo con el que forme colocación. Si tomamos, por ejemplo, el nombre CHANCE
} 
lengua y necesitamos, por consiguiente, que el diccionario realice esta función. Es evidente la importancia que esta herramienta tiene, tanto para la comunidad lingüística, especialmente para los traductores, como para el hablante en general y, por este motivo, lo hemos considerado digno objeto de estudio.

Entre los diferentes tipos de diccionarios nos centraremos en los monolingües, ya que su especificidad semántica es distinta de la de los bilingües en la medida en la que se utiliza el léxico de una sola lengua. Esta característica permite establecer relaciones semánticas y morfológicas dentro de una misma lengua sin tener que entrar en las equivalencias con otros idiomas. No podemos olvidar, por otra parte, que los diccionarios monolingües también constituyen una de las herramientas imprescindibles para la traducción, además de un elemento primordial en el estudio de una segunda lengua.

Los diccionarios elegidos para el estudio son Longman Dictionary of Contemporary English (LDOCE) y Collins COBUILD English Language Dictionary (COBUILD). Esta selección ha tratado de recoger dos de las editoriales más representativas de la lexicografía inglesa dirigida a estudiantes de segunda lengua, ciñéndose a dos diccionarios por motivos de espacio. Tanto el $L D O C E$ como el COBUILD están comprendidos en un solo tomo y sus usuarios potenciales son estudiantes de inglés como L2 para los que la información sobre colocaciones es de suma importancia. Pero ¿son conscientes los editores de esta importancia?

Para comprobar cómo estos diccionarios se han enfrentado a esta cuestión y cómo la han resuelto, hemos realizado una búsqueda restringida al campo de la combinatoria léxica. Dentro de este campo hemos investigado, en concreto, las colocaciones que sigan la regla de Mel'cuk (1998: 29), cuyos elementos sean un verbo light (Jespersen 1954: 117) o verbo funcional (Wotjak 1998: 259) seguido de un artículo indeterminado y un nombre, siendo éste último el que llevaría la carga léxica. Dichas construcciones analíticas suelen alternar con construcciones sintéticas realizadas a través de un verbo simple homógrafo y, hasta cierto punto, sinónimo. Por ejemplo,

(1) to have a shower $=>$ to shower,

(2) to make a remark $=>$ to remark,

(3) to take a sip $=>$ to sip.

La coexistencia de ambas formas nos anima a ampliar la búsqueda de información sobre estas colocaciones también al artículo del verbo simple. Por motivos de espacio localizaremos información sobre las construcciones de este tipo que se puedan realizar con los verbos take, have y make. Por consiguiente, se realizará una triple búsqueda dirigida a las entradas de estos tres verbos funcionales, a las entradas de los nombres con los que forman una colocación y a las entradas de los verbos simples.

del Oxford Collocations Dictionary $(O C D)$, comprobaremos que incluye cuatro lexemas diferentes con una combinatoria léxica particular. Como muestra incluiremos algunos de estos lexemas con sus sentidos, los verbos con los que pueden formar colocación y los ejemplos del $O C D$ :

CHANCE ' 'possibility' (give, have), the doctors gave him little chance of surviving the night,

CHANCE $E_{2}$ 'opportunity' (have, take), I finally have the chance to meet my hero,

CHANCE 'risk' (take), the guide book didn't mention the hotel, but we decided to take a chance. 
La estructura del trabajo será la siguiente:

El punto 2 se centrará en el tipo de combinatoria léxica y concepto de colocación utilizado para nuestro estudio. En el punto 2.1 presentaremos las partes de una colocación según su carga semántica. Este tema será muy importante para el posterior desarrollo del trabajo, ya que los criterios lexicográficos deberían basarse en esta relación jerárquica. En el punto 3 se presentarán los resultados del estudio de los dos diccionarios, comenzando por el COBUILD 3.1, siguiendo con el LDOCE 3.2. Por último, el punto 4 recogerá las conclusiones extraídas de la investigación.

\section{LA NOCIÓN DEL TÉRMINO "COLOCACIÓN"}

El término "colocación" es entendido de muy diferentes maneras según los autores (Alonso Ramos 1994-95; Corpas Pastor 1996; Cowie 1998; Koike 2001; Bosque 2001), pero, por motivos de espacio, no entraremos en este trabajo en la discusión de los diferentes significados de este término, sino que presentaremos y adoptaremos la propuesta de Hausmann (1985), Benson, Benson \& Ilson (1986, 1997) y Mel'cuk (1995, 1998). Apoyándonos en estos autores y ciñéndonos a la clasificación de Live (1973), nos centraremos en la representación lexicográfica de un tipo determinado de colocaciones léxicas en los dos diccionarios elegidos COBUILD y $L D O C E$.

Los diccionarios no deben incluir combinaciones libres de palabra o, como las denomina Hausmann (1985:120), combinaciones banales, es decir, aquéllas que se pueden formar gracias a los conocimientos que el hablante posee sobre el léxico y la gramática; sin embargo, sí deben hacer algo más que describir palabras aisladas, incluyendo también información sobre combinatoria léxica restringida. En ese apartado es donde debería incluirse la descripción de nuestras colocaciones (Mel'cuk 1995), dado que esta información forma parte del significado de un determinado lexema (Firth 1957: 196; Alonso Ramos 2002). Los usuarios del diccionario necesitan este tipo de información para realizar tanto las tareas de codificación como de decodificación de una lengua. Por consiguiente, las editoriales tendrían que prestar especial atención a este punto.

La gran mayoría de los estudios lingüísticos centrados en la rama de la lexicografía abogan por incluir en los diccionarios la información necesaria que facilite al usuario las pautas de cómo las palabras se combinan para formar oraciones. En casi todas las lenguas, o quizás en todas, existen una serie de construcciones fijas, no idiomáticas y, hasta cierto punto, fácilmente identificables por un hablante nativo. A estos grupos de palabras los denominan Benson, Benson \& Ilson (1986; 1997: xv) recurrent combinations (combinaciones recurrentes), fixed combinations (combinaciones fijas) o collocations (colocaciones). El grupo de las colocaciones lo dividen en dos subgrupos, grammatical collocations (colocaciones gramaticales) $^{3}$ y lexical collocations (colocaciones léxicas).

En sentido general Hausmann (1985: 119) se refiere con el término "colocación” a las limitaciones en la coocurrencia de dos lexemas donde éstos no se combinan libremente, sino que uno determina semánticamente al otro. De manera más explícita y formal, Mel'cuk

\footnotetext{
${ }^{3}$ Dentro de este subgrupo se incluirían las colocaciones que constan de una palabra dominante (nombre, adjetivo, verbo) y una preposición o estructura gramatical tal como un infinitivo o una cláusula. Como ejemplos encontramos: aim at, account for, angry at, entre otras.
} 
(1998: 29) considera colocaciones aquellas combinaciones de lexemas que lleguen a cumplir la regla o fórmula que incluimos en el siguiente cuadro:

$\mathbf{A B}=<^{\prime} \mathrm{A} \rho^{4} \mathrm{C}^{\prime} ; / \mathrm{A} \rho \mathrm{B} />\mid$ ' $\mathrm{C}$ ' is expressed by $\mathbf{B}$ such that /A $\rho$ B/ is not constructed unrestrictedly ${ }^{5}$

Los diferentes análisis a los que han sido sometidas las colocaciones léxicas ponen de manifiesto que están a medio camino entre las combinaciones libres y las locuciones idiomáticas. No llegan a ser libres porque uno de los lexemas determina de alguna manera la aparición del otro, pero al mismo tiempo no son locuciones idiomáticas, porque el significado final sí que refleja el significado de alguna de las partes. Hemos elegido el estudio de este tipo de colocaciones porque, en comparación con las colocaciones gramaticales, son más difíciles de identificar y la controversia sobre el modo en el que deben ser representadas en los diccionarios es mayor.

Dentro de las colocaciones léxicas nos centraremos en el caso Ia de Mel'cuk (1998: $31)^{6}$, pero de este grupo elegiremos sólo las colocaciones que entran dentro del tipo I de Live (1973: 35), cuya estructura sería la siguiente:

\begin{tabular}{|l|l|l|l|}
\hline verbo semivacío & $a$ & nombre deverbal & sufijo derivativo $\imath$ \\
\hline
\end{tabular}

y que puedan ser sustituidas por un verbo simple que sea homógrafo y sinónimo del nombre (siempre que la construcción no forme una locución idiomática ${ }^{7}$ ). Esta caracte-

\footnotetext{
${ }^{4}$ El significado de este símbolo, denominado operation of linguistic union, se encuentra en Mel'cuk (1998: 27).

${ }^{5}$ Mel'cuk las denomina también semi-frasemas y explica esta regla de la siguiente manera:

A COLLOCATION AB of language $\boldsymbol{L}$ is a semantic phraseme of $\boldsymbol{L}$ such that its signified ' $X$ ' is constructed out of the signified of one of its two constituent lexemes-say, of $A-$ and a signified ' $C$ ' [' $X$ ' $=$ ' $A \rho C$ '] such that the lexeme $\boldsymbol{B}$ expresses ' $C$ ' only contingent on $\boldsymbol{A}$ (Mel'cuk 1998:30).

$\mathrm{AB}$ tendría un significante /A $\rho$ B/ y dos posibles significados, 'B $\rho$ C' o 'A $\rho$ C', dependiendo de cual de los dos elementos sea la base de la colocación.

${ }^{6} \mathrm{La}$ regla anterior representa, para Mel'cuk, los cuatro tipos más importantes de colocaciones. Si consideramos que el significado de ' $\mathrm{C}$ ' es distinto al significado de 'B', nos encontraremos con dos posibilidades:

Ia) ' $C$ ' es, por decirlo de alguna manera, un significado vacío (esto convierte al lexema $\mathbf{B}$ en una especie de semiauxiliar seleccionado por $\mathbf{A}$ como soporte o apoyo de la configuración sintáctica; p.ej. to do a favour, to give a look, to take a step);

Ib) 'C' no está vacío de significado, pero el lexema $\mathbf{B}$ expresa 'C' sólo cuando acompaña a A o lexemas del mismo campo semántico que A (p.ej. black coffee, French window).

$\mathrm{Si}$, por el contrario, el significado de 'C' es igual al significado de 'B', podemos tener también dos posibilidades:

IIa) que el significado 'B' no pueda ser expresado con A de ninguna otra manera (p. ej. strong coffee (*powerful coffee), heavy rain (*weighty rain);

IIb) que el significado de 'B' incluya una parte del significado de 'A' (p.ej. aquiline nose, blond hair, rancid butter, donde no podemos explicar el significado de aquiline, blond o rancid sin hacer referencia a nariz, pelo y mantequilla).

${ }^{7}$ Como podría ser el caso de to take/have a hand (in) con el sentido de 'be involved in' o to give something a whirl 'try something' o to take a bath 'to lose a lot of money on an investment' (COLLINS COBUILD 1995).
} 
rística nos permite observar si la colocación puede aparecer bajo la entrada de $\mathrm{V}_{0}$, de $\mathrm{N}_{1}$ o $\mathrm{V}_{1}^{8}$.

Este tipo de unidades ofrece unas limitaciones tanto desde el punto de vista del elemento literal como del utilizado en sentido figurado. Así, por ejemplo, podemos encontrar have (como elemento figurado) en una serie de combinaciones restringidas y con una serie de nombres limitados. A modo de ejemplo tenemos, entre otros, los nombres incluidos en las colocaciones (4), (5) y (6); sin embargo, el ejemplo (7) no sería correcto (Wierzbicka 1982, Dixon 1992) ${ }^{9}$.

(4) to have a shower,

(5) to have a walk,

(6) to have a drink $k^{10}$,

(7) *to have an eat.

¿Cómo y dónde debería incluírse esta información en los diccionarios? Antes de pasar a este punto es necesario hablar de los elementos que componen una colocación para identificar los espacios que el lexicógrafo tiene a su disposición.

\subsection{Elementos de la colocación}

Es evidente que una colocación bimembre posee dos elementos bajo los cuales podemos introducirla. El problema descriptivo más importante que presentan este tipo de combinaciones léxicas (además de los señalados por Cowie (1981: 232 ${ }^{14}$ ) es elegir el lugar donde se introducirá la información en el diccionario. La elección no debe basarse en una mera variación metalingüística, sino que tiene que deberse a la aplicación de una teoría gramatical, consiguiendo así una unidad de criterios $^{12}$.

\footnotetext{
${ }^{8} \mathrm{~V}_{0}$ se utiliza para nuestros verbos soporte (have, take, make) $\mathrm{N}_{1}$ para el nombre deverbal. En to have a walk tendríamos $\mathrm{N}_{1}=$ walk, $\mathrm{V}_{0}=$ have. Mientras que $\mathrm{V}_{1}$ se utilizaría para la construcción sintética realizada a través del verbo simple to walk.

${ }^{9}$ Stein (1991) critica, de alguna manera, las explicaciones de Wierzbicka y Dixon. Sus argumentos para aclarar por qué algunos nombres no pueden formar parte de estas colocaciones se basan en que el espacio de ciertos sentidos ya está ocupado por otros lexemas. En el ejemplo (7) el sentido que podría tener el nombre eat está ya ocupado por meal o snack, que sí pueden formar parte de esta colocación (to have a meal, to have a snack).

${ }^{10}$ Los verbos que participan en estas construcciones reciben una acentuación débil, al igual que los auxiliares, en vez del acento fuerte que recae sobre los correspondientes verbos plenos (Live 1973). Basándose en este última característica, Live aduce que la expresión he had several drinks no es una construcción de verbo frasal, sino el verbo have seguido de su complemento directo. Las diferencias entre he had a drink y he had several drinks estribarían tanto en el acento, que sería débil en el caso del verbo frasal (had a drink), como en la imposibilidad de sustituir had several drinks por el verbo simple to drink. ${ }^{11}$ Como serían, primero mostrar el grado de variación que puede existir entre dos colocaciones que pertenezcan a la misma clase gramatical. Por ejemplo, entre by comparison y by definition la primera admite by/in comparison (with sb/sth), mientras que la segunda sólo se puede utilizar con una preposición. La segunda sería la variación en las funciones sintácticas de los miembros de una determinada clase o frase y su representación. Entre los ejemplos de Cowie se encuentran las frases preposicionales by the hour, by all means y by comparison, que realizan diferentes funciones dentro de la oración.

12 Actualmente se está llevando a cabo un proyecto en la Universidad de A Coruña bajo la dirección de Margarita Alonso Ramos, que consiste en la creación de un diccionario de colocaciones del español
} 
Para discutir acerca de dónde debe introducirse esta información, debemos examinar antes las diferentes partes de las que consta una colocación. Entre los dos miembros de una colocación existe un determinado estatus que viene dado por la relación de jerarquía que se establece entre los elementos. Así pues, dado que uno de los miembros determina al otro, Hausmann (1985: 119) propone la denominación de Basis (base) para el que determina y Kollokator (colocativo) para el que es determinado. Siguiendo también este razonamiento, Benson, Benson \& Ilson (1997: ix) denominan a la palabra que determina dominant word (palabra dominante) y dan una serie de pautas para saber cuál sería el elemento dominante en su clasificación de las colocaciones ${ }^{13}$.

La elección de uno u otro elemento como lugar de inclusión de la información debe seguir unos criterios fijos y previos a la creación del diccionario. La postura de Hausmann (1985: 121-122) a este respecto se basa en la función para la que se destine el diccionario, bien codificación o decodificación. Según este autor, si el diccionario está destinado a la decodificación (comprensión o recepción de textos), la colocación debe introducirse bajo la entrada del colocativo; ahora bien, si su función es la de codificación (producción o creación de textos), la colocación debe situarse bajo la base. Retomando este razonamiento, pero dado que la mayoría de los diccionarios no están pensados exclusivamente para una u otra función, Hausmann propone los principios de la semiotaxis ${ }^{14}$ en 1997. Siguiendo estos principios estaría claro que este tipo de información debería de introducirse en las bases (nombres) y no en los colocativos (verbos). Por otra parte, Hausmann (1999: 206) considera que la eliminación de la información colocacional de las entradas de los colocativos puede ser un procedimiento demasiado revolucionario, pero totalmente lógico si nos basamos en los principios de la semiotaxis.

Otra posible opción es duplicar la información. Este procedimiento puede resultar un problema, sobre todo para los diccionarios en papel debido a su limitado espacio. Aunque esta limitación, no obstante, desaparece en cierto modo en los diccionarios informatizados, creemos que es mejor utilizar notas de referencia en los diccionarios convencionales e hipertexto en los informatizados. Defendemos, por consiguiente, la postura de recoger la información siempre bajo la base, tanto en el caso de la codificación como de la decodificación de textos. Creemos que para la producción de textos es totalmente imprescindible, y en el caso de la decodificación no supone ninguna ayuda encontrarse unos artículos como los reservados a los verbos have, make etc. en los que la información se esconde entre numerosas acepciones. En cualquier caso, aunque utilicemos la entrada del colocativo, siempre tendremos que hacer referencia a la base, ya que, desde el punto de vista de la semiotaxis, el colocativo es una palabra sinsemántica. Esto quiere decir que sólo adquiere ese significado concreto cuando va acompañando a la base y, por tanto, sólo puede ser definido haciendo referencia a ésta, que sería, en contraposición, autosemántica.

(DICE). En esta dirección (http://www.dicesp.com/) se pueden observan con absoluta claridad las pautas y la meticulosidad con que éstas se siguen en el proyecto.

${ }^{13}$ Si hay un nombre en la colocación, será el nombre; si hay dos nombres, será el segundo nombre; si no hay nombre, será el adjetivo y si no hay nombre ni adjetivo, será el verbo.

${ }^{14}$ Hausmann (1997: 172) la define como "la dimensión semántica de la sintagmática" que se ocupa de "las posibilidades definitorias de las palabras". 


\section{ESTUDIO DE LAS OPCIONES LEXICOGRÁFICAS}

Una vez que hemos aclarado el concepto de colocación en el que nos basaremos y las partes que la componen, veamos qué política han seguido los diccionarios que hemos seleccionados para el análisis (COBUILD y $L D O C E)$.

El proceso de trabajo ha sido el siguiente: primeramente buscar en las entradas de los verbos funcionales take, have y make (en su función de colocativo) y tratar de localizar información sobre colocaciones con take (8), have (9) y make (10), del estilo de las siguientes:

(8) take a walk / bath/swim / sip / dip,

(9) have a talk / read / cough / sleep / try,

(10) make a call / comment / remark/ turn /start etc.

Seguidamente se ha buscado esta misma información en las entradas de las bases (los nombres en este caso) y finalmente, en los verbos simples.

\subsection{Comportamiento del Collins COBUILD English Language Dictionary (COBUILD)}

Empezaremos por analizar los verbos take, have y make para hacer una breve descripción de las entradas.

Las entradas de estos verbos comienzan hablando de su uso en expresiones en las que el verbo no tiene un significado propio, sino que el significado se extrae del nombre que lo acompaña (su objeto directo) o, expresado de otra manera, explican su carácter general y su casi completa delexicalización. Seguidamente se introduce una lista con los posibles grupos de nombres a los que se puede unir el verbo y sus múltiples sentidos (más de 40 en los tres casos). Uno de estos sentidos tiene siempre un espectro tan amplio que permitiría la construcción de cualquier colocación. Por ejemplo, dentro de este diccionario encontramos las siguientes acepciones:

take 1.10 with a large number of other nouns to form expressions referring to actions, activities or events of some kind.

have 7.1 used with nouns that describe actions.

make 1.5 with many other nouns to perform expressions which take most of their meaning from the noun.

La excesiva generalización y la compresión de tanta información dificultan nuestra tarea de localización de estas colocaciones verbales. Los campos semánticos de los nombres son tan generales o su descripción tan vaga, que casi cualquier nombre podría aparecer en combinación con estos verbos. En la práctica todas las colocaciones que hemos buscado han podido ser adaptadas a uno u otro de los sentidos incluidos en la entrada del verbo funcional.

Este exceso de generalización nos puede llevar a confusiones como sobreentender del sentido 1.1 de take (" with nouns that refer to physical actions ") que la colocación to run 
a race 'correr una carrera' podría realizarse también con el verbo take. Sin embargo, según los datos del BBI y del propio COBUILD, la colocación * take a race no es correcta. También, siguiendo estas definiciones, se podría crear una colocación como *make a job, cuando la colocación correcta, hoy en día, es do a job.

Una vez examinados los colocativos nos dirigimos a las entradas de los nombres, encontrando resultados muy diversos. Este diccionario opta por no introducir en las bases información sobre estas colocaciones de una manera directa y resaltada. Si bien es verdad que hemos podido localizar la mayoría de estos verbos funcionales en las entradas de los nombres, el procedimiento lexicográfico seguido opta por recogerlos en diferentes apartados. En algunos casos se localizan a través del contexto (frases con situaciones hipotéticas que normalmente utiliza el COBUILD); en otros artículos se opta por la sección de los ejemplos, probablemente la más utilizada para esta información colocacional; pero también es posible localizarlos en ambos a la vez. En la entrada del nombre bath tenemos una muestra de inclusión en los dos apartados:

bath $[\ldots] 2$ When you have or take a bath or when you are in the bath, you wash your body while sitting or lying in a bath filled with water. EG I'm going to have a bath... ${ }^{15}$

Llama la atención en la entrada de este nombre que la colocación to run a bath aparezca en negrilla como sentido 5 de bath y que no se siga la misma política para have I take a bath donde sólo se pone en negrilla la base (el nombre).

Como excepciones a la regla anterior podemos mencionar las colocaciones have a read/ cough y make a start, a las que no se hace ninguna referencia en la entrada de los nombres, ni a través de los ejemplos ni del contexto. En estos casos pudimos comprobar que las propias colocaciones aparecen como ejemplos en los sentidos $\mathbf{7 . 4}$ y $\mathbf{2 7}$ de have y 5 de make respectivamente.

Los problemas que plantea el introducir las colocaciones en la sección de los ejemplos (la preferida por este diccionario) es que, al ser el número de ejemplos limitado, nos podemos encontrar con artículos como el de swim. En la entrada de este nombre sólo se recoge una colocación dentro de la sección de los ejemplos, en concreto go for a swim, pero no aparece ni have ni take a swim. ¿Debemos suponer entonces que no serían construcciones correctas de la lengua o, por el contrario, acogernos al punto $\mathbf{1 . 1}$ de take para su formación? Si optamos por la segunda opción, como ya hemos visto anteriormente, corremos el riesgo de construir un gran número de combinaciones incorrectas.

Otro de los problemas que plantea este diccionario es la creación de artículos excesivamente largos. Esto se debe a la introducción de información sobre las diferentes clases de palabras homógrafas u homónimas en la misma entrada, dificultando la búsqueda y oscureciendo los resultados. Por ejemplo, encontramos algunos casos en los que dentro del mismo párrafo en el que se define el verbo simple se hace referencia a su utilización como nombre y, seguidamente, se introduce un ejemplo que incluye el verbo funcional que debe acompañar a este nombre. Como muestra incluiremos uno de estos casos, el del

${ }^{15}$ El subrayado es mío. 
artículo de smoke (muestra 1), aunque este procedimiento se ha seguido con más colocaciones, como por ejemplo have a turn/ a try/ a talk y make a turn.

\begin{tabular}{|l|r|}
\hline 3 If you smoke a cigarette, pipe, etc, & V or V + O \\
you have it in your mouth, sucking the & \\
smoke [...]. EG [...] he sat and smoked & \\
and stared out of the window. <used as & \\
a noun. EG I'm dying for a smoke... & \\
We went outside to have a smoke. & \\
\hline
\end{tabular}

Muestra 1: artículo de smoke

Se puede decir que, en estos casos, la información aparece en la entrada del verbo simple, aunque señalizando su uso como nombre en esta construcción (N SING: a+N). Sin embargo, no será hasta el sentido 5 cuando encontraremos la entrada del nombre $(5 \mathrm{~A}$ smoke is $[\ldots])$, por tanto tendremos todavía un sentido verbal, el 4 (4 If you smoke, [...]), entre esta colocación y la entrada del nombre.

Veamos, por último, un ejemplo de otras de las opciones elegidas por este diccionario para recoger este tipo de colocaciones. Las colocaciones elegidas serán make a call y make a comment. En la siguiente tabla se observará la información colocacional que hemos localizado bajo el colocativo y bajo la base:

\begin{tabular}{|c|c|c|}
\hline Colocación & Colocativo & Base \\
\hline \multirow{2}{*}{ make a call } & make & call \\
\hline & $\begin{array}{l}2 \text { if you make a telephone call, you } \\
\text { telephone someone. EG He had two } \\
\text { phone calls to make [...] }\end{array}$ & $\begin{array}{l}9.1 \text { a communication that you receive } \\
\text { or make by telephone. EG [...]I made } \\
\text { a long-distance call to Aberdeen. }\end{array}$ \\
\hline \multirow[b]{2}{*}{$\begin{array}{l}\text { make a } \\
\text { comment }\end{array}$} & make & comment \\
\hline & $\begin{array}{l}1.1 \text { with nouns that express speech } \\
\text { actions. EG }[. . .] \text { he made no } \\
\text { comment... }\end{array}$ & $\begin{array}{l}2 \text { a comment is a statement of } \\
\text { opinion about something. EG People } \\
\text { in the town started making rude } \\
\text { comments. }\end{array}$ \\
\hline
\end{tabular}

Muestra 2: información en las bases y en los colocativos

A la vista de las muestras 1 y 2, la lista de los caminos elegidos por el COBUILD para recoger información colocacional sobre este tipo de colocaciones verbales con verbo funcional es la siguiente: 


\begin{abstract}
XLa colocación puede aparecer como parte del contexto introducido en la situación hipotética (muestra 2: if you make a call, que está localizado en la entrada de make),

Xcon información general sobre el campo semántico de los nombres con los que puede formar colocación: with nouns that express speech actions (bajo make, muestra 2),

Xtambién es posible encontrarla en la sección de los ejemplos (en la muestra 2 aparece en las cuatro entradas),

Xcon una llamada de atención sobre su uso como nombre en la entrada del verbo simple (como en el caso de smoke, muestra 1), introduciéndolo a través del ejemplo (we went outside to have a smoke).
\end{abstract}

No obstante, no todas las colocaciones de este tipo han sido introducidas en el diccionario. También existe la posibilidad de que la información sobre la colocación se haya omitido y no aparezca en ninguno de los lugares anteriormente mencionados.

Se puede comprobar que no se sigue un criterio lexicográfico específico para presentar esta información. Por otro lado, cada entrada recoge demasiada información que se amalgama sin un método tipográfico que facilite el reconocimiento e identificación de la información requerida. Además, los casos en los que se generaliza la información, como en la muestra 2 (with nouns that express speech actions) pueden dan lugar a errores de uso. Por ejemplo, si consideramos, lógicamente, que el nombre question pertenece a este campo semántico, podríamos, siguiendo esta información, formar la incorrecta colocación *make a question.

Por último, si analizamos el diccionario desde el punto de vista semiotáctico (Hausmann 1997), veremos que el COBUILD sigue los principios semiotácticos, que proponen que las palabras sinsemánticas se definan después de introducir el contexto o cotexto, dado que necesitamos primeramente conocer la base (o palabra autosemántica) para poder crear una definición y no al revés. Si nos centramos, por ejemplo, en las entradas de nuestros verbos funcionales, veremos que este diccionario sigue estos principios. Como podemos observar a continuación, en la entrada $\mathbf{2 3}$ del verbo make, se introducen primero unas posibles bases (co-texto) y seguidamente, a la derecha, la definición:

$$
\text { co-texto } \mid \text { definición }
$$

23 If you make a visit, trip, or journey, you go or visit somewhere [...].

\title{
3.2. Comportamiento del Longman Dictionary of Contemporary English (LOCDE)
}

El estudio de este diccionario, siguiendo los mismos pasos que en el apartado anterior, presenta los siguientes resultados en lo concerniente a estructura y contenido:

Del verbo take se extraen 11 apartados, entre sentidos y posibles usos, donde se utilizan etiquetas tan generales como el punto 2 Do something o el 10 Other meanings. Dentro del punto $\mathbf{2}$ se describe el verbo como sigue:

2 A word meaning to do something, used with many different nouns to form a phrase that means 'do the action connected with the noun' [...]. 
Una descripción más o menos parecida se obtiene en las entradas de los verbos have y make, variando únicamente el número de sentidos que se otorga a éstos (49 para have y 15 para make). La información extra que incluye este diccionario en el apartado de los verbos se refiere a su frecuencia de utilización en el lenguaje hablado o escrito. El procedimiento utilizado es el siguiente:

$\mathrm{X}$ bien se añade un gráfico, como en la entrada de take, donde se puede ver, en este caso, que el verbo es más utilizado en el lenguaje hablado ${ }^{16}$,

$\mathrm{X}$ bien se introducen unas abreviaturas ( $\mathrm{S}$ para spoken y W para written) seguidas de 1,2 o 3, según sea el porcentaje de aparición en ambos registros en el British National Corpus y el Longman Lancaster Corpus.

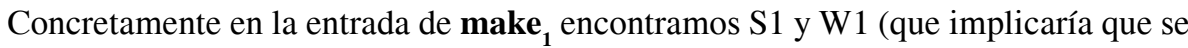
utiliza por igual en ambos registros), mientras que en la de have $\mathbf{2}_{\mathbf{2}}$ localizaremos sólo S1, resaltando su uso más frecuente en el lenguaje hablado.

La investigación en las entradas de los nombres arroja resultados más clarificadores y sigue una política de presentación que resulta de más ayuda para el usuario. En casi todos los casos analizados las colocaciones con verbo funcional han sido recogidas en las entradas de los nombres, en negrilla y separadas por una barra vertical de la definición. Por ejemplo,

walk $n \mathbf{1}[\mathrm{C}]$ a journey that you make by walking, especially for exercise or enjoyment: it's a long walk. Maybe we should get the bus. | go for a walk [...] | take / have a walk [...].

Sólo en algunos casos como have a drink / a read / a sleep / a cough o make a turn la colocación se introduce a través de un ejemplo en la entrada del nombre, mientras que en otros, como have a swim, se reenvía desde $\operatorname{swim}_{n}$ a las notas de utilización ${ }^{17}$ de bath. El reenvío a otras entradas no sigue unas pautas preestablecidas y consistentes en los artículos de nombres que presentan un cierto grado de sinonimia. Por ejemplo, la colocación take / have a dip se presenta dentro del nombre dip en vez de reenviarla a las notas de uso de bath, donde también aparece. Por último, hemos encontrado un caso (have a smoke) en el que la colocación aparece sólo bajo el colocativo, presentándola como sentido 5 de la entrada de have : $_{2}$

$5<$ EAT /DRINK /SMOKE = to eat, to drink, or to smoke: she sat down and had another drink. |have lunch / a meal etc. I usually have breakfast at 8 o'clock.

La subentrada está organizada de la siguiente manera: primeramente aparecen los sentidos generales a los que se refiere el verbo have cuando va acompañado de algunos de

\footnotetext{
${ }^{16}$ Las notas de uso de este diccionario suponen una información adicional extensa sobre la utilización de los diferentes lexemas e introduce, además, las diferencias de uso entre el inglés americano y el inglés británico.

${ }^{17}$ Estas notas de utilización incluyen información semántica, pragmática o de combinatoria léxica sobre lexemas del mismo campo semántico.
} 
los nombres que pertenecen a esos campos semánticos. El problema que constatamos aquí es que si bien los dos últimos pueden hacer también función de co-texto con el verbo have (have a drink, have a smoke), el primero hace referencia sólo al campo semántico de la comida y no a la posible construcción *have an eat (Wierzbicka 1982). Seguidamente, se utiliza la forma sintética como paráfrasis definitoria de la colocación y, después de los ejemplos, se añaden otras posibles colocaciones con nombres que pertenecen a ese mismo campo.

Veamos una muestra de cómo se puede localizar una de estas colocaciones en este diccionario:

\begin{tabular}{|c|c|c|}
\hline Colocación & Colocativo & Base \\
\hline & make $_{1}$ & call $_{2}$ \\
\hline make a call & $\begin{array}{l}15 \text { OTHER MEANINGS } \\
37 \text { make a (phone) call to speak to } \\
\text { someone using a telephone: I have } \\
\text { to make a few calls. }\end{array}$ & $\begin{array}{l}n \mathbf{1}<\text { TELEPHONE }=[\mathrm{C}] \text { a) an } \\
\text { attempt to speak to someone by } \\
\text { telephone }[. . .] \mid \text { make a call It's } \\
\text { cheaper to make calls after 6pm. }\end{array}$ \\
\hline
\end{tabular}

Muestra 3: información en la base y en el colocativo

Comprobamos que la información se presenta claramente con un método tipográfico fácilmente reconocible. No sólo el nombre, como en el caso del COBUILD, sino la colocación completa, se resalta en negrilla en ambas entradas (muestra 3). Además, un gran número de estas colocaciones ha sido recogido por este diccionario, prefiriendo la bases para introducir información colocacional (Lareo Martín 2004).

Desde el punto de vista semiotáctico, el LDOCE cumple también los principios expuestos por Hausmann (1997). La política adoptada opta por definir las palabras sinsemánticas, introduciendo primero el co-texto que les debe acompañar para adquirir un determinado significado. Esta información precede a la definición, siguiendo así los principios de la semiotaxis.

\section{CONCLUSIONES}

Las corrientes actuales se decantan por incluir en los diccionarios toda la información precisa, tanto en el plano de combinatoria gramatical como de combinatoria léxica, de modo que el usuario (hablante nativo, traductor, estudiante) sepa cómo combinar las palabras para construir frases.

La información sobre colocaciones es de suma importancia para cualquier tipo de usuarios de una lengua, por eso creemos que los lexicógrafos deberían seguir un método tipográfico para resaltar de una manera especial estas peculiaridades al igual que lo hacen con las locuciones idiomáticas. Esta política sería de gran ayuda a la hora de conocer el grado de combinabilidad restringida de las palabras y facilitaría la tarea y tiempo de acceso a la información. 
En la oferta lexicográfica que hemos analizado, dirigida a estudiantes de una segunda lengua para los que estos datos son de suma importancia, se puede decir que, en general, resulta difícil localizar este tipo de información. Hemos visto la tendencia que muestran los dos diccionarios a introducir las colocaciones en la sección de los ejemplos, opción que, en nuestra opinión dificulta su búsqueda y limita el número de inclusiones. La inclusión de dos o tres ejemplos no puede aclarar las dudas que se plantearan sobre otras colocaciones hipotéticas, puesto que no se puede extraer un patrón lógico del comportamiento de este tipo de combinaciones.

A la vista de los resultados del estudio podemos decir que el COBUILD, a pesar de ser el que más datos ofrece, recoge menos información sobre este tema que el LDOCE. La presentación de la información en el COBUILD se amalgama en una sola entrada para las diferentes clases de palabras homógrafas, resaltando en negrilla, en las diferentes acepciones, la palabra cabeza del artículo en vez del co-texto. Por el contrario, en el LOCDE la presentación es más clara y de más ayuda para el usuario. Aquí vemos cómo la colocación entera está resaltada en negrilla y separada por una barra vertical de la definición (...| make a call). Además, se incluyen datos sobre la frecuencia de utilización en los diferentes registros, también muy útiles para el usuario.

En resumen, el $L O C D E$ es el diccionario más satisfactorio en lo concerniente a la localización de este tipo de colocaciones. Sin embargo, ninguno de los dos diccionarios sigue una política clara y uniforme. Hemos comprobado cómo algunas de las colocaciones no aparecen recogidas en ninguna entrada, otras pueden encontrarse bajo el colocativo y bajo la base, y otras, indistintamente, en uno de los dos.

Dentro de la polémica sobre el lugar en el que debería introducirse este tipo de información y constatando las diferentes posturas que siguen los diccionarios (incluso al tratar el mismo tipo de colocaciones), nosotros nos sumamos a la opinión de Hausmann (1999: 206). Su postura nos parece una manera más satisfactoria de resolver el problema. Al seguir esta vía, es decir, actuando regidos por los principios de la semiotaxis, definiremos sólo las palabras autónomas o autosemánticas en su propia entrada en orden alfabético. Esto quiere decir, que, como sólo el nombre tendrá una entrada en el diccionario, este tipo de colocaciones deberían recogerse bajo el nombre. Si además tenemos en cuenta que el significado de estos verbos en combinación con algunos nombres está léxicamente determinada por el significado del nombre, la localización más lógica para la definición de estos verbos colocativos sería bajo dicho nombre. Los verbos que entran en estas colocaciones son palabras sinsemánticas en su papel de verbo funcional; por este motivo no es lógico introducir su significado funcional en la entrada del verbo.

Si abordamos el problema desde otro punto de vista, nos encontramos con que el significado colocacional de estos verbos podría considerarse parte de la polisemia de estos verbos en su estatus de verbo funcional. En este caso deberían incluirse las colocaciones bajo la entrada del verbo. Sin embargo, debido a la cantidad de significados diferentes que pueden adquirir estos verbos dependiendo del co-texto, creemos que no sería de mucha ayuda suministrar una enorme lista de posibles bases bajo el colocativo. Terminaremos, por tanto, apoyando la postura que elige la base o palabra autosemántica para introducir esta información colocacional tanto en los diccionarios destinados a la codificación como a la decodificación. 


\section{REFERENCIAS}

Alonso Ramos, M. 1994-1995. "Hacia una definición del concepto de colocación: de J. R. Firth a I. A. Mel'cuk”. Revista de Lexicografía 1: 9-28.

. 2002. "Colocación y contorno de la definición lexicográfica". Lingüística Española Actual XXIV/1: 63-96.

Benson, M., E. Benson \& R. Ilson. 1986. The Lexicographic Description of English. Amsterdam/ Philadelphia: John Benjamins Publishing.

1997. The BBI Dictionary of English Word Combinations. Amsterdam/ Philadelphia: John Benjamins Publishing.

Bosque, I. 2001. "Sobre el concepto de colocación y sus límites”. Lingüística Española Actual XXIII/1: 9-40.

CowIE, A. P. 1981. "The Treatment of Collocations and Idioms in Learners' Dictionaries". Applied Linguistics 2/3: 223-235.

Ed. 1998. Phraseology: Theory, Analysis and Applications. Oxford/New York: Oxford University Press.

Diccionario de colocaciones del español (DICE). http://www.dicesp.com/ (Mayo 2005).

Dixon, R. M. W. 1992. A New Approach to English Grammar on Semantic Principles. Oxfrod: Clarendon Press.

FIRTH, J. R. 1957. "Modes of Meaning”. Papers in Linguistics 1934-1951. London: Oxford University Press, 190-215.

Hausmann, F. J. 1985. "Kollokationen im deutschen Wörterbuch: ein Beitrag zur Theorie des lexikographischen Beispiels". Lexikographie und Grammatik. Akten des Essener Kolloquiums zur Grammatik im Wörterbuch. Eds. H. BergenHolTz \& J. Mugdon. Lexicographica. Series Maior 3. Tübingen: Max Niemeyer Verlag, 118129.

. 1997. "Semiotaxis und Wörterbuch". Linguistische Theorie und lexikographische Praxis. Eds. K. P. Konerding y A. LeHr. Lexicographica Series Maior 82. Tübingen: Max Niemeyer Verlag, 171-179.

1999. "Semiotaxis and Learners' Dictionaries". The Perfect Learners' Dictionary (?). Eds. T. Herbst \& K. Popp. Tübingen: Max Niemeyer Verlag, 205211.

Jespersen, O. 1954 (1942). A Modern English Grammar on Historical Principles. Part VI Morphology. With the assistance of P. Christophersen, N. Haislund \& K. Schibsbye. London: George Allen \& Unwin.

KoIKe, K. 2001. Colocaciones léxicas en el español actual: estudio formal y léxicosemántico. Alcalá de Henares: Servicio de publicaciones, Universidad de Alcalá. 
LAREO MARTín, I. 2004. "La representación de algunas colocaciones verbales inglesas en los diccionarios y su comparación con el Dictionnaire explicatif et combinatoire du français contemporain (DEC)". Actas del V Congreso de Lingüística General II. Ed. M. VillayandRe Llamazares. Madrid: ARCO/LIBROS, 1693-1702.

Live, A. H. 1973. "The TAKE-HAVE Phrasal in English". Linguistics 95: 31-50.

Longman Dictionary of Contemporary English (LDOCE). 1995. Harlow: Longman.

MeL'cuk, I. 1988. "Semantic Description of Lexical Units in an Explanatory Combinatorial Dictionary: Basic Principles and Heuristic Criteria". International Journal of Lexicography 1/3: 165-188.

1998. "Collocations and Lexical Functions". Phraseology: Theory, Analysis and Applications.Ed. A. P. CowIE. Oxford/ New York: Oxford University Press, 2353.

1995. "Phrasemes in Language and Phraseology in Linguistics". Idioms: Structural, and Psychological Perspectives. Eds. M. EvERAERT, E. VAN DER Linden, A. Schenk \& R. Schreuder. New Jersey: Lawrence Erlbamm Associates, 167-232.

Oxford Collocations Dictionary. 2002. Oxford: Oxford University Press.

Sinclair, J. ed. 1993. Collins COBUILD English Language Dictionary. (COBUILD). London: HarperCollins.

1995. Collins COBUILD Dictionary of Idioms. London: HarperCollins.

Stein, G. 1991. “The Phrasal Verb Type 'to Have a Look' in Modern English”. International Review of Applied Linguistics in Language Teaching, 29: 1-29.

WierzBickA, A. 1982. "Why can you have a drink when you can't *have an eat". Language, 58: 753-799.

Wotjak, G. 1998. "Reflexiones acerca de construcciones verbo-nominales funcionales". Estudios de fraseología y fraseografía del español actual. Ed. G. Wотנак. Madrid: Iberoamericana, 257-279. 